\title{
Brownian Motion with Restoring Drift: The Petit and Micro-Canonical Ensembles
}

\author{
H. P. McKean, K. L. Vaninsky \\ CIMS, 251 Mercer St., New York, NY 10012, USA
}

Received: 31 March 1993

Abstract: Let $f(Q)$ be odd and positive near $+\infty$. Then the non-linear wave equation $\partial^{2} Q / \partial t^{2}-\partial^{2} Q / \partial x^{2}+f(Q)=0$, considered on the circle $0 \leq x<L$, can be written in Hamiltonian form $Q^{\bullet}=\partial H / \partial P, P^{\bullet}=-\partial H / \partial Q$ with

$$
P=Q^{\bullet} \quad \text { and } \quad H=\frac{1}{2} \int_{0}^{L}\left(Q^{\prime}\right)^{2}+\int_{0}^{L} F(Q)+\frac{1}{2} \int_{0}^{L} P^{2}
$$

the corresponding flow preserves the (suitably interpreted) "petit ensemble" $e^{-H} d^{\infty} Q d^{\infty} P$; and, for $L \uparrow \infty, Q$ settles down to the stationary diffusion with infinitesimal operator $\frac{1}{2} \partial^{2} / \partial Q^{2}+m(Q) \partial / \partial Q, m$ being the logarithmic derivative of the ground state of $-d^{2} / d Q^{2} \mid F(Q)$. This diffusion is the "Brownian motion with restoring drift"; see McKean-Vaninsky [1993(1)]. For reasons suggested by the paper of Lebowitz-Rose-Speer [1988] on NLS, it is interesting to study the "micro-canonical ensemble" obtained by restricting to the sphere $\int_{0}^{L} Q^{2}=N$ and making $L \uparrow \infty$ with fixed $D=N / L$. Now, for $F(Q) / Q^{2} \rightarrow \infty$, the same type of diffusion appears, but with drift arising from the modified potential $F(Q)+c Q^{2}, c$ being chosen so that the mean of $Q^{2}$ is the assigned number $D$. The proof employs Döblin's method of "loops" [1937] and steepest descent. The same is true for $F(Q)=m^{2} Q^{2}$, only now the proof is elementary. The outcome is also the same if $F(Q) / Q^{2} \rightarrow 0$, provided $D$ is smaller than the petit canonical mean of $Q^{2}$; for $D$ larger than this mean, the matter is more subtle and the outcome is unknown.

This work was performed at the Courant Institute of Mathematical Sciences, with the partial support of the National Science Foundation under NSF Grant NO. DMS-9112664 which is gratefully acknowledged by H. P. McKean 


\section{Introduction}

Let $f(Q)$ be odd and positive near $+\infty$ and let $F(Q)=\int_{0}^{Q} f$. The recipe

$$
d M_{L}=\exp \left\{-\int_{-L / 2}^{L / 2} F[Q(x)] d x\right\} \times \frac{\exp \left\{-\frac{1}{2} \int_{-L / 2}^{L / 2}\left[Q^{\prime}(x)\right]^{2} d x\right\}}{(2 \pi 0+)^{\infty / 2}} d^{\infty} Q
$$

defines a measure on the space of continuous paths $x \rightarrow Q(x)$ of period $L$. The second factor is "circular" Brownian measure, obtained by conditioning the "standard" Brownian motion so that $Q(L / 2)=Q(-L / 2)$ and distributing this common value $h$ over the line according to the infinite measure $(2 \pi L)^{-1 / 2} \times d h$, i.e., if $E_{00}$ is the "tied" Brownian mean and if $I(Q)$ is any reasonable function of the path, then

$$
\int I(Q) \frac{\exp \left\{-\frac{1}{2} \int\left(Q^{\prime}\right)^{2}\right\}}{(2 \pi 0+)^{\infty / 2}} d^{\infty} Q=\int_{-\infty}^{\infty} E_{00}[I(Q+h)] \frac{d h}{\sqrt{2 \pi L}} .
$$

The first factor is a mere density; it controls the partition function $Z_{L}=$ the total mass, the latter being finite or infinite together with $\int_{0}^{\infty} e^{-L F(h)} d h$. This type of measure figures in the (petit) canonical ensemble for the classical wave equation $\partial^{2} Q / \partial t^{2}-$ $\partial^{2} Q / \partial x^{2}+f(Q)=0$ : in fact, with the Hamiltonian $H=\frac{1}{2} \int P^{2}+\frac{1}{2} \int\left(Q^{\prime}\right)^{2}+\int F(Q)$, you have $Q^{\bullet}=P=\partial H / \partial P$ and $P^{\bullet}=Q^{\prime \prime}-f(Q)=-\partial H / \partial Q$, and the canonical measure

$$
e^{-H} d \mathrm{vol}=e^{-\int F(Q)} \frac{\exp \left\{-\frac{1}{2} \int P^{2}\right\}}{(2 \pi 0+)^{\infty / 2}} d^{\infty} P \times \frac{\exp \left\{-\frac{1}{2} \int\left(Q^{\prime}\right)^{2}\right\}}{(2 \pi 0+)^{\infty / 2}} d^{\infty} Q
$$

is invariant under the flow, suitably interpreted; see McKean-Vaninsky [1993(1)].

$Z_{L}<\infty$ for $L \uparrow \infty$ is now assumed, and $M_{L}$ is re-expressed in terms of a Brownian motion with (restoring) drift. $Z_{L}<\infty$ implies $F( \pm \infty)=\infty$, so $\mathfrak{G}_{0}=-(1 / 2) d^{2} / d Q^{2}+F(Q)$ has pure point spectrum $\lambda_{0}\left(\mathfrak{G}_{0}\right)<\lambda_{1}\left(\mathfrak{G}_{0}\right)<$ etc. $\uparrow \infty$ and (positive) ground state $\psi_{0}$ with $\int \psi_{0}^{2}(Q) d Q=1$, say. Let $m$ be the logarithmic derivative of $\psi_{0}$, compute $d \lg \psi_{0}[Q(x)]$ in the Brownian manner:

$$
\begin{aligned}
d \lg \psi_{0}[Q(x)] & =\left(\lg \psi_{0}\right)^{\prime}(Q) d Q+\frac{1}{2}\left(\lg \psi_{0}\right)^{\prime \prime}(Q)(d Q)^{2} \\
& =m(Q) d Q-\frac{1}{2} m^{2}(Q) d x+\left[F(Q)-\lambda_{0}\left(G_{0}\right)\right] d x,{ }^{1}
\end{aligned}
$$

and integrate from $-L / 2$ to $+L / 2$, noting that $\int d \lg \psi_{0}=0$. It follows that

$$
\begin{aligned}
d M_{L}= & \exp \left\{\int m(Q) d Q-\frac{1}{2} \int m^{2}(Q) d x\right\} \\
& \times \frac{\exp \left\{-\frac{1}{2} \int\left(Q^{\prime}\right)^{2}\right\}}{(2 \pi 0+)^{\infty / 2}} d^{\infty} Q
\end{aligned}
$$

$\overline{1(d Q)^{2}=d x}$, in accord with Itô's lemma: in fact, under the conditioning $Q(L)=Q(0)$, $Q(x)=B(x)-(x / L) B(L)$ with a standard Brownian motion $B$ 
up to a numerical factor which is dropped, and here you recognize the law of the (circular) diffusion with infinitesimal operator $\mathfrak{G}=(1 / 2) \partial^{2} / \partial Q^{2}+m(Q) \partial /$ $\partial Q,{ }^{2}$ conditioned as before so that $Q(L / 2)=Q(-L / 2)$, this common value being distributed over the line according to the finite measure $p(L, h, h) d h .^{3}$ The total mass is now

$$
Z_{L}=\int_{-\infty}^{\infty} p(L, h, h) d h=\sum_{n=0}^{\infty} e^{\lambda_{n}(\mathfrak{G}) L}
$$

with $\lambda_{n}(\mathfrak{G})=-\lambda_{n}\left(\mathfrak{G}_{0}\right)+\lambda_{0}\left(\mathfrak{G}_{0}\right)$, as appears from the similarity $-\psi_{0} \mathfrak{G} \psi_{0}^{-1}=$ $G_{0}-\lambda_{0}\left(\mathfrak{G}_{0}\right)$, and from $\lambda_{0}(\mathfrak{G})=0$ it is plain that $Z_{L}=1+o(1)$ for $L \uparrow \infty$. The drift $m(Q)$ is odd and negative for large $Q>0$, i.e., it pulls $Q$ back to rest $(Q=0)$, and it comes as no surprise that, for $L \uparrow \infty, M_{L}$ tends to the laws $M_{\infty}$ of the stationary diffusion with infinitesimal operator $G$ and invariant distribution $\psi_{0}^{2}(Q) d Q ;{ }^{4}$ see McKean-Vaninsky [1993(1)].

The same ideas apply to the nonclassical wave equation NLS ( = cubic Schrödinger) for $L<\infty$. Now $\sqrt{-1} \partial Q / \partial t=-\partial^{2} Q / \partial x^{2} \pm|Q|^{2} Q$ with Hamiltonian $H=\int\left|Q^{\prime}\right|^{2} \pm \frac{1}{2} \int|Q|^{4},{ }^{5}$ and the canonical measure

$$
e^{-H} d \mathrm{vol}=e^{\mp(1 / 2) \int|Q|^{4}} \frac{\exp \left\{-\int\left|Q^{\prime}\right|^{2}\right\}}{(2 \pi 0+)^{\infty}} d^{\infty}(\text { real } Q) d^{\infty}(\text { imag } Q)
$$

is invariant under the flow, ${ }^{6}$ with this difference: that, with the lower (focussing) sign, $Z_{L}=\infty$. This divergence prompted Lebowitz-Rose-Speer [1988] to introduce the micro-canonical ensemble, obtained by conditioning the canonical measure upon a fixed value $N$ of the constant of motion $\int|Q|^{2}$, and to speculate that the temperaturedependent micro-canonical ensemble with $e^{-H / T}$ in place of $e^{-H}$ might exhibit a phase transition in the thermodynamic limit as $L \uparrow \infty$ with fixed $D=N / L$, favoring solitons/radiation at low/high temperature. Their program is in its infancy and it seemed profitable to make a trial run with the canonical measure $M_{L}$ introduced at the start, artifically conditioned upon $\int Q^{2}=N$. Denote this micro-canonical ensemble by $M_{N / L=D}$ and take the thermodynamic limit, as above. The result is as predicted by Gibb's principle of equivalence of ensembles (maximal entropy production): as $L \uparrow \infty$ with fixed $D=N / L$, the micro-canonical ensemble $M_{N / L=D}$ approaches the petit ensemble $M_{\infty}^{*}$ based upon $F^{*}(Q)=F(Q)+c Q^{2}$, the number $c$ being adjusted so that $M_{\infty}^{*}(Q)^{2}=D$. The rest of the paper is mostly occupied by the proof of this fact under the (more or less) necessary condition that $Q^{-2} F(Q)$ tends to $+\infty$ with $Q$, but see Sect. 2 which deals with the special case $F(Q)=m^{2} Q^{2} / 2$.

What happens for $Q^{-2} F(Q)=o(1)$ is, in part, more subtle. The recipe stands with a suitable choice of $c \geq 0$ if $D \leq M_{\infty}\left(Q^{2}\right)$, but if $D>M_{\infty}\left(Q^{2}\right)$ it cannot be so. Then $c$ could only be negative and $F^{*}(Q)=c Q^{2}[1+o(1)]$ acts as a repulsive force far out; as such, it is incapable of producing a stationary diffusion, so while the microcanonical ensemble makes sense and its thermodynamic limit surely exists, something else must come out. The same problem arises in an aggravated form, for $\mathrm{KdV}$ with

\footnotetext{
2 This is the Cameron-Martin formula [1945] as adapted to diffusions by Girsanov [1960]; see, for example, McKean [1969]

${ }^{3} p$ is the transiton density of the diffusion, i.e., it is the (smooth) elementary solution of $\partial p / \partial x=\mathfrak{G} p$

${ }^{4} \mathfrak{G}^{\ddagger} \psi_{0}^{2}=0$

$5 \sqrt{-1} Q^{\bullet}=\partial H / \partial Q^{*}$ with $*=$ conjugation

6 McKean-Vaninsky [1993(2)]
} 
$F(Q)=-Q^{3}$, and for focussing NLS with $F(Q)=-Q^{4}$ and a 2-dimensional Bessel process in place of the Brownian motion. Both cases fall naturally into the micro-canonical format, $\int Q^{2}$ being a constant of motion, and the micro-canonical ensemble makes sense since $E\left[\exp \int Q^{6-} \mid \int Q^{2}=N\right]<\infty,{ }^{7}$ but the outcome of the thermodynamic limit is unknown.

The present condition $Q^{-2} F(Q) \uparrow \infty$ permits a simple attack (steepest descent) capitalizing upon the diffusion format expounded above and an allied method (loops) of Döblin [1937]. The next section (2) presents the explicitly solvable example $F(Q)=m^{2} Q^{2} / 2$; it is a continuous analog of the spherical model of Berlin-Kac [1952], for which see also the finer results of Molchanov-Sudarev [1975]. Section 3 is preparatory to the main computation (Sect. 4). Section 5 isolates the local limit theorem which lies at the bottom of it all and places it in the context of Martin boundaries. Gibbs' principle occupies the final section (6); compare, esp. Kolmogorov [1949] and also Dobrushin-Tirozzi [1977] for its novel insistence upon the fact that, for Gibbsian-Markovian lattice fields, the local central limit theorem and its superficially weaker integral form are really one and the same.

\section{An Example}

The micro-canonical ensemble for the Ornstein-Uhlenbeck process with mass $m$ provides the simplest illustration; it corresponds to $F(Q)=m^{2} Q^{2} / 2$. The petit partition function is found to be

$$
Z=\int \frac{\exp \left\{-\frac{1}{2} \int\left[\left(Q^{\prime}\right)^{2}+m^{2} Q^{2}\right]\right\}}{(2 \pi 0+)^{\infty / 2}} d^{\infty} Q=\frac{1}{2 \operatorname{sh}(m L / 2)} .
$$

Fix a compact test function $\phi$ and, to evaluate the micro-canonical mean ${ }^{8}$

$$
M_{N / L=D}\left[e^{\int \phi Q}\right]=\frac{M_{L}\left[e^{\int \phi Q}, \int Q^{2}=N\right]}{Z_{N / L=D}=M_{L}\left[\int Q^{2}=N\right]},
$$

take the transform

$$
\begin{aligned}
& \int_{0}^{\infty} e^{-c^{2} N / 2} M_{L}\left[e^{\int \phi Q}, \int Q^{2}=N\right] d N \\
& \quad=Z_{+}^{-1} \int \frac{e^{-(1 / 2) \int\left[\left(Q^{\prime}\right)^{2}+m_{+}^{2} Q^{2}\right]}}{(2 \pi 0+)^{\infty / 2}} e^{\int \phi Q} d^{\infty} Q \times Z_{+} \quad \text { with } m_{+}^{2}=m^{2}+c^{2} \\
& =e^{(1 / 2) \int \phi K \phi} \times \frac{1}{2 \operatorname{sh}\left(m_{+} L / 2\right)}
\end{aligned}
$$

in which $K$ is the (periodic) Green's function for $-d^{2} / d x^{2}+m_{+}^{2}$, and invert it with respect to the variable $s=c^{2} / 2$ to obtain

$$
M_{N / L=D}\left[e^{\int \phi Q}\right]=Z_{N / L=D}^{-1} \frac{1}{2 \pi \sqrt{-1}} \int_{-i \infty}^{+i \infty} \frac{e^{s N} e^{(1 / 2) \int \phi K \phi}}{2 \operatorname{sh}\left(m_{+} L / 2\right)} d s
$$

\footnotetext{
7 See Lebowitz-Rose-Speer [1988]

$8 M(I)$ and the like are expectations $M(I)=\int I d M ; M\left[\int Q^{2}=N\right]=(\partial / \partial N) M\left[\int Q^{2} \leq N\right]$ and the like stand for densities
} 
Now comes the descent:

$$
\frac{e^{s N}}{2 \operatorname{sh}\left(m_{+} L / 2\right)}=\frac{e^{L\left(s D-(1 / 2) \sqrt{m^{2}+2 s}\right)}}{1-e^{-L \sqrt{m^{2}+2 s}}}
$$

is analytic in the $s$-plane cut from $-\infty$ to $-m^{2} / 2$, and the integral is controlled by the contribution from the critical point $s=1 / 8 D^{2}-m^{2} / 2$ of function $s D-\frac{1}{2} \sqrt{m^{2}+2 s}$ figuring in the top exponent, ${ }^{9}$ with the result that

$$
\lim _{L \uparrow \infty} M_{N / L=D}\left[e^{\int \phi Q}\right]=e^{\frac{1}{2} \int \phi K \phi}
$$

where $K$ is now the whole-line Green's funtion for $-d^{2} / d x^{2}+m_{*}^{2}$ with $m_{*}=1 / 2 D$, i.e., the thermodynamic limit simply changes the mass from $m$ to $m_{*}=1 / 2 D$. This agrees with the statement of Sect. 1: for mass $m, \psi_{0}^{2}(Q)=(m / \pi)^{1 / 2} e^{-m Q^{2}}$ and $\int \psi_{0}^{2} Q^{2}=1 / 2 m$ so that mass $1 / 2 D$ produces mean square $D$. The genereal computation is, naturally, more difficult; it is carried out in the next two sections under the stated condition $Q^{-2} F(Q) \uparrow \infty$.

\section{Preliminaries}

The restriction of the petit measure to the micro-canonical ensemble and the implementation of the thermodynamic limit require a few preparations, both general and technical.

Generalities. The first task is to explain why the conditioning by the value of $\int Q^{2}=N$ makes sense. $x \rightarrow Q(x)$ is the diffusion with infinitesimal operator $\mathfrak{G}=(1 / 2) \partial^{2} / \partial Q^{2}+m(Q) \partial / \partial Q$. The motion $x \rightarrow\left[Q(x), I(x)=\int_{0}^{x} Q^{2}\right]$ is also a diffusion, but now its infinitesimal operator $\mathfrak{G}_{+}=\mathfrak{G}+Q^{2} \partial / \partial I$ is degenerate in that $\partial^{2} / \partial I^{2}$ is missing from the top of it. This is not troublesome: the double commutator $\left[\partial / \partial Q,\left[\partial / \partial Q, Q^{2} \partial / \partial I\right]\right]=2 \partial / \partial I$ reproduces the missing vector field and now a deep theorem of Hörmander [1967] guarantees that the joint density 10 $p(x, A, B, I)=P\left[Q(x)=B, \int_{0}^{x} Q^{2}=I \mid Q(0)=A\right]$ is smooth in all its variables and also positive, provided only that $x>0 .{ }^{11}$ This fact dispels any anciety as to the propriety of the micro-canonical ensemble: for example, if $0<x_{1}<x_{2}<L$, then the micro-canonical measure of the event $\left(a_{1} \leq Q\left(x_{1}\right)<b_{1}\right) \cap\left(a_{2} \leq Q\left(x_{2}\right)<b_{1}\right)$ is nothing but

$$
\begin{aligned}
& Z^{-1} \int_{-\infty}^{+\infty} d Q_{0} \int_{a_{1}}^{b_{1}} d Q_{1} \int_{a_{2}}^{b_{2}} d Q_{2} \int_{I_{1}+I_{2} \leq N} d^{2} I \\
& \times p\left(x_{1}, Q_{0}, Q_{1}, I_{1}\right) p\left(x_{2}-x_{1}, Q_{1}, Q_{2}, I_{2}\right) p\left(L-x_{2}, Q_{2}, Q_{0}, N-I_{1}-I_{2}\right)
\end{aligned}
$$

$9\left(s D-\frac{1}{2} \sqrt{\text { etc. }}\right)^{\prime \prime}=1 / 16 D^{3}>0$ at the critical point; the line of integration is bent so as to pass through the latter

$10 P\left[Q(x)=Q, \int_{0}^{x} Q^{2}=I\right]$ means $(\partial / \partial Q)(\partial / \partial I) P\left[Q(x) \leq Q, \int_{0}^{x} Q^{2} \leq I\right]$. This kind of unconventional but handy notation is used throughout

11 Krylov [1987] can be consulted for such matters 
with normalizer

$$
Z=\int_{-\infty}^{\infty} p(L, Q, Q, N) d Q
$$

Now the statement to be proved is that, for any nice function $H(Q)$ depending upon a limited sample of the path, such as $Q(x): 0 \leq x \leq 1$, the micro-canonical mean

$$
M_{N / L=D}(H)=Z^{-1} \int_{-\infty}^{\infty} E_{Q}\left[H, Q(L)=Q, \int_{0}^{L} Q^{2}=N\right] d Q
$$

tends, as $L \uparrow \infty$ with fixed $N / L=D$, to the mean

$$
M_{\infty}^{*}(H)=\int_{-\infty}^{\infty}\left[\psi_{0}^{*}(Q)\right]^{2} d Q E_{Q}^{*}(H)
$$

for the stationary diffusion with infinitesimal operator $\mathfrak{G}^{*}$ arising from $F^{*}(Q)=$ $F(Q)+c Q^{2}$, the number $c$ being adjusted so that $M_{\infty}^{*}\left(Q^{2}\right)=\int\left[\psi_{0}^{*}(Q)\right]^{2} Q^{2}$ takes the prescribed value $D .{ }^{12}$ Naturally, it is necessary to check that this adjustment can really be made. The fact is plain if $F(Q)=m^{2} Q^{2} / 2$ since $M_{\infty}\left(Q^{2}\right)=1 / 2 m$, as noted before. Now consider the general case $Q^{-2} F(Q) \uparrow \infty$. For $c \uparrow \infty$, the ground state $\psi_{0}$ for $F^{*}(Q)=F(Q)+c Q^{2}$ satisfies

$$
c \int Q^{2} \psi_{0}^{2} \leq \frac{1}{2} \int\left(\psi_{0}^{\prime}\right)^{2}+\int F^{*}(Q) \psi_{0}^{2} \leq \frac{1}{2} \int\left(\psi^{\prime}\right)^{2}+\int F(Q) \psi^{2}+c \int Q^{2} \psi^{2}
$$

for any nice function $\psi$ with $\int \psi^{2}=1$, from which it appears that $D=\int Q^{2} \psi_{0}^{2}$ can be made as small as you like: just concentrate $\psi$ in the vicinity of $Q=0$. Contrariwise for $c \downarrow-\infty$ the same appraisal shows that $\int Q^{2} \psi_{0}^{2}$ can be made as big as you like by spreading $\psi^{2}$ out. Now pick $c$ so that $\int Q^{2} \psi_{0}^{2}=D$ and note that the micro-canonical ensemble is not changed by the adjustment $F(Q) \rightarrow F(Q)+c Q^{2}$ : because of the conditioning $\int Q^{2}=N$, it washes out above and below. This means that the proof needs to be made only for $c=0$, i.e., when $N / L=D$ is already the mean $M_{\infty}\left(Q^{2}\right)$ for the original petit ensemble.

Idea of the Proof. This is easy to describe if $M_{\infty}\left(Q^{2}\right)=D$. Let $H$ depend upon $Q(x): 0 \leq x \leq 1$, say. Then

$$
\begin{aligned}
M_{N / L=D}(H)= & Z^{-1} \int_{-\infty}^{\infty} E_{Q}\left[H, Q(L)=Q, \int_{0}^{L} Q^{2}=N\right] d Q \\
= & Z^{-1} \int_{-\infty}^{\infty} d Q \int_{-\infty}^{\infty} d Q^{\prime} \int_{0}^{N} d I E_{Q}\left[H, Q(1)=Q^{\prime}, \int_{0}^{1} Q^{2}=I\right] \\
& \times P_{Q^{\prime}}\left[Q(L-1)=Q, \int_{0}^{L-1} Q^{2}=N-I\right] .
\end{aligned}
$$

$\overline{12 \int \psi_{0}^{2} Q^{2}<\infty}$ as soon as $Q^{-2} F(Q) \uparrow \infty$ in view of $\int\left(\psi_{0}^{\prime}\right)^{2}+\int F(Q) \psi_{0}^{2}=\lambda_{0}\left(\mathfrak{G}_{0}\right)$ 
It is to be shown that, for $L \uparrow \infty$ with fixed $N / L=D$,

$$
P_{Q^{\prime}}\left[Q(L)=Q, \int_{0}^{L} Q^{2}=N\right]
$$

is proportional to $L^{-1 / 2} \psi_{0}^{2}(Q) \times[1+o(1)]$, with the result that

$$
\begin{aligned}
M_{N / L=D}(H) & \cong \frac{\int_{-\infty}^{\infty} d Q \int_{-\infty}^{\infty} d Q^{\prime} \int_{0}^{\infty} d I E_{Q}\left[H, Q(1)=Q^{\prime}, \int_{0}^{1} Q^{2}=I\right] \psi_{0}^{2}(Q)}{\int_{-\infty}^{\infty} d Q \int_{-\infty}^{\infty} d Q^{\prime} \int_{0}^{\infty} d I P_{Q}\left[Q(1)=Q^{\prime}, \int_{0}^{1} Q^{2}=I\right] \psi_{0}^{2}(Q)} \\
& =M_{\infty}(H) .
\end{aligned}
$$

Let $L_{0}$ be the passage time from $Q(0)=Q^{\prime}$ to $Q=0$ and let $L_{1}>L_{0}, L_{2}>L_{1}$ etc. be the successive "loop times" for passing from $Q=0$ to $Q=1$ and back; see Fig. 1.

Fig. 1
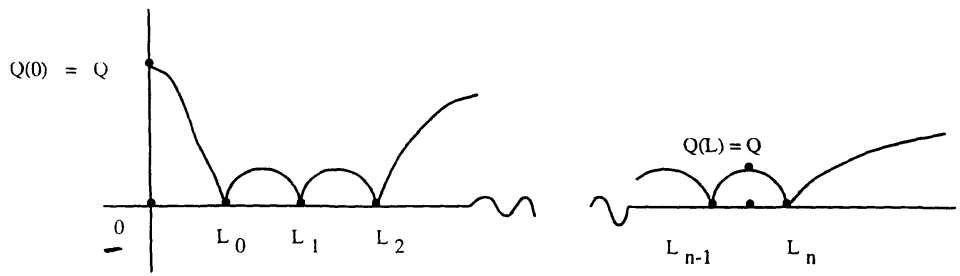

The individual loops $Q(x): L_{n-1} \leq x \leq L_{n}$ are independent and identically distributed;

$$
D=M_{\infty}\left(Q^{2}\right)=\lim _{L \uparrow \infty} L^{-1} \int_{0}^{L} Q^{2}=\lim _{n \uparrow \infty} L_{n}^{-1} \int_{0}^{L_{n}} Q^{2}=\frac{E_{0}\left(\int_{0}^{L_{1}} Q^{2}\right)}{E_{0}\left(L_{1}\right)},
$$

by the law of large numbers; and the diffusion settles (exponentially fast) into its stationary regime, so it is natural to hope that

$$
\begin{aligned}
& P_{Q^{\prime}}\left[Q(L)=Q, \int_{0}^{L} Q^{2}=N\right] \\
& \quad=P_{Q^{\prime}}\left[\int_{0}^{L} Q^{2}=N \mid Q(L)=Q\right] \times P_{Q^{\prime}}[Q(L)=Q] \\
& \cong P_{Q^{\prime}}\left[\int_{0}^{L} Q^{2}=N\right] \times \psi_{0}^{2}(Q),
\end{aligned}
$$


in which the first factor simplifies itself, in response to the law of large numbers and the (local) central limit theorem, as follows:

$$
\begin{aligned}
P_{Q^{\prime}}\left[\int_{0}^{L} Q^{2}=N\right] & =P_{Q^{\prime}}\left[\sum_{k=1}^{n} \int_{L_{k-1}}^{L_{k}}\left(Q^{2}-D\right)=0\right] \text { with } n=\left[L / E_{0}\left(L_{1}\right)\right] \\
& =P_{Q^{\prime}}\left[\frac{1}{\sqrt{n}} \sum_{k=1}^{n} \int_{L_{k-1}}^{L_{k}}\left(Q^{2}-D\right)=0\right] \frac{1}{\sqrt{n}} \\
& \cong \frac{1}{\sqrt{2 \pi \sigma^{2} n}} \text { with } \sigma^{2}=E_{0}\left(\int_{0}^{L_{1}} Q^{2}-D L_{1}\right)^{2},
\end{aligned}
$$

i.e.,

$$
P_{Q^{\prime}}\left[Q(L)=Q, \int_{0}^{L} Q^{2}=N\right] \cong \frac{1}{\sqrt{2 \pi \sigma^{2} L / E_{0}\left(L_{1}\right)}} \times \psi_{0}^{2}(Q)
$$

It remains to hope, in addition, that the shift $L \rightarrow L-1$ and $N \rightarrow N-I$ will not disturb this appraisal, and it is the content of Sect. 4 that this is perfectly correct, but be warned: if the adjustment $M_{\infty}\left(Q^{2}\right)=D$ is not made beforehand, the present method predicts that $c=-\Delta D / 2 \sigma^{2}$, and this is not correct as it takes $c \rightarrow \infty$ to make $D \downarrow 0$ - in short, the conditioning $Q(L)=Q$ must now distort the Gaussian law, and that is not so easy to track.

Technicalities. These are needed in Sect. 4; at a first reading, just note the facts and pass on. $Q(0)=0, T$ is the passage time to $1, Q$ is a free variable, and $\mathfrak{t}(x, Q)$ is the local time $\lim _{\varepsilon \downarrow 0} \varepsilon^{-1}$ meas $\left(0 \leq x^{\prime} \leq x: Q \leq Q\left(x^{\prime}\right)<Q+\varepsilon\right){ }^{13}$

Item 1. $E_{0}\left(e^{r T}\right)<\infty$ for small values of $r>0$.

Proof. $\mathfrak{G}$ has pure point spectrum and it is the same if you kill the particle at $Q=1$, only now the ground state is displaced from $\lambda_{0}(\mathfrak{G})=0$ to $c<0$. The rest follows from

$$
\begin{aligned}
P_{0}(T=x) & =-(\partial / \partial x) \int_{-\infty}^{1} p(x, 0, Q) d Q=-\int_{-\infty}^{1} \mathfrak{G}^{\ddagger} p(x, 0, Q) d Q \\
& =-\left.\left[\frac{1}{2} \frac{\partial}{\partial Q}-m(Q)\right] p(x, 0, Q)\right|_{-\infty} ^{1} \\
& =-\frac{1}{2} p_{3}(x, 0,1) \leq \text { constant } \times e^{c x},
\end{aligned}
$$

$p$ being the transition density for the killed diffusion. ${ }^{14}$

13 See Itô-McKean [1965] for such matters

14 See Itô-McKean [1965] for details 
Item 2. $E_{0}[\mathfrak{t}(T, Q)]=\int_{Q^{+}}^{1} \psi_{0}^{-2} \times 2 \psi_{0}^{2}(Q)$ for $Q \leq 1, Q^{+}$being the larger of $Q$ and 0; also $E_{0}\left[\mathfrak{t}^{2}(T, Q)\right]=\int_{Q^{+}}^{1} \psi_{0}^{-2} \int^{1} \psi_{0}^{-2} \times \psi_{0}^{4}(Q)$.

Proof. $E_{0}(\mathfrak{t})=\int_{0}^{\infty} p(x, 0, Q) d x$ is nothing but the Green's function $G(0, Q)$ for the operator $\mathfrak{G}^{15}$ restricted to $Q \leq 1$, with killing at $Q=1$, and from $\mathfrak{G}=$ $(1 / 2) \psi_{0}^{-2}(\partial / \partial Q) \psi_{0}^{2}(\partial / \partial Q)$, it is easy to see that

$$
G\left(Q^{\prime}, Q\right)=\int_{0}^{1} \psi_{0}^{-2} \times 2 \psi_{0}^{2}(Q)
$$

"spot" being the larger of $Q$ and $Q^{\prime}$. The second evaluation is similar.

Item 3. $E_{0}\left[\int_{0}^{T} x d \mathfrak{t}(x, Q)\right] \leq \int_{0}^{1} \psi_{0}^{-2} \int^{1} \psi_{0}^{-2} \int_{-\infty}^{1} 2 \psi_{0}^{2} \times 2 \psi_{0}^{2}(Q)$.

Proof. $E_{0}$ (etc.) $=\int_{0}^{\infty} x p(x, 0, Q)=\int_{-\infty}^{1} G\left(0, Q^{\prime}\right) G\left(Q^{\prime}, Q\right) d Q^{\prime}$; now use the explicit form of $G$ from Item 2 .

Item 4. ${ }^{16} E_{0}\left[\int_{0}^{T} I(x) d \mathfrak{t}(x, Q)\right] \leq \int_{0}^{1} \psi_{0}^{-2} \int^{1} \psi_{0}^{-2} \int_{-\infty}^{1} Q^{2} 2 \psi_{0}^{2} \times 2 \psi_{0}^{2}(Q)$.

Proof. See Item 3.

Item 5. $E_{0}\left[e^{-\alpha T-\beta I(T)}\right]$ is of modulus $<1$ for $\alpha$ and $\beta$ in the closed (right-hand) half-plane, the origin expected; moreover, it vanishes as $\alpha$ and/or $\beta$ tend to $\infty$ in that region.

Proof. The expectation can be of modulus 1 only if both $\alpha$ and $\beta$ are real and $P_{0}[\alpha T+\beta I \in 2 \pi Z]=1 .{ }^{17}$ But a typical path starting at 0 and hitting 1 for the first time will immediately overshoot, so that if $Q$ is such a path with $\alpha T+\beta I=2 \pi n$, then it is the same for any nearby path. But this is manifestly wrong if it would happen with positive probability. The rest follows from the deeper fact that $T$ and $I=I(T)$ have a joint density: indeed, $h(Q)=E_{Q}\left[e^{-\alpha T-\beta I}\right]$ satisfies $\mathfrak{G} h=\left(\alpha+\beta Q^{2}\right) h$ for $Q<1$, which is to say that the (possibly fictitious) density $p=P_{Q}[T=x, I=N]$ is a weak solution of $\partial p / \partial x=\mathfrak{G} p-Q^{2} \partial p / \partial N$, and now Hörmander [1967] guarantees that $p$ is an honest function.

\footnotetext{
$15 \mathfrak{G} G=-1$

$16 I(x)=\int_{0}^{x} Q^{2}$

$17 Z$ is now the integers
} 


\section{The Thermodynamic Limit}

Fix a nice function $H$ depending upon $Q(x): 0 \leq x \leq 1$, say, and form its microcanonical mean

$$
\begin{aligned}
M_{N / L=D}(H)= & Z^{-1} \int E_{Q}\left[H, Q(L)=Q, \int_{0}^{L} Q^{2}=N\right] d Q \\
= & Z^{-1} \int d Q \int d Q^{\prime} \int_{0}^{N} d N^{\prime} \\
& \times E_{Q}\left[H, Q\left(L^{\prime}\right)=Q^{\prime}, \int_{0}^{-L^{\prime}} Q^{2}=N^{\prime}\right] \\
& \times P_{Q^{\prime}}\left[Q\left(L-L^{\prime}\right)=Q, \int_{0}^{L-L^{\prime}} Q^{2}=N-N^{\prime}\right]
\end{aligned}
$$

with $1 \leq L^{\prime} \leq 2<L$, say. Now the micro-canonical mean of $Q^{2}(x)$ is $D=N / L$, independently of $0 \leq x<L,{ }^{18}$ so only a small error is incurred if the integration is restricted to a big box in $Q Q^{\prime} N^{\prime}$-space; in particular, it is permitted to replace the $Q Q^{\prime}$-density in the top of the micro-canonical mean by the double convolution

$$
\begin{aligned}
I * I I= & \int_{0}^{L} d L^{\prime} \int_{0}^{N} d N^{\prime} E_{Q}\left[H, Q\left(L^{\prime}\right)=Q^{\prime}, \int_{0}^{L^{\prime}} Q^{2}=N^{\prime}\right] A\left(L^{\prime}\right) B\left(N^{\prime}\right) \\
& \times P_{Q^{\prime}}\left[Q\left(L-L^{\prime}\right)=Q, \int_{0}^{L-L^{\prime}} Q^{2}=N-N^{\prime}\right]
\end{aligned}
$$

in which $A$ and $B$ are smooth compact functions, with $A$ vanishing outside $[1,2]$, $\int_{1}^{2} A=1$, and $B=1$ on a long interval containing $N^{\prime}=0$.

The introduction of $A$ and $B$ and the integration over $1 \leq L^{\prime} \leq 2$ looks artificial but is essential to the method. It makes $I$ smooth and compact, with transform $\widehat{I}=\int_{0}^{\infty} \int_{0}^{\infty} e^{-\alpha L^{\prime}-\beta N^{\prime}} I d L^{\prime} d N^{\prime}$ which is both analytic in $C^{2}$ and class $C_{\downarrow}^{\infty}\left(\sqrt{-1} R^{2}\right)$. This decay, or something like it, must be present to justify the descent: without it, the inverse transform simply masks the realities; moreover, it cannot be obtained from $\widehat{I I}$, as is easily confirmed for the case of pure Brownian motion: in fact, with positive

$18 M_{N / L=D}\left[Q^{2}(x)\right]=L^{-1} \int_{0}^{L} M\left[Q^{2}\left(x^{\prime}\right)\right] d x^{\prime}=L^{-1} M\left[\int_{0}^{L} Q^{2}\right]=N / L=D$ 
imaginary $\alpha=\sqrt{-1} p$ and $\beta=\sqrt{-1} q / 2$,

$$
\begin{aligned}
& \int_{0}^{\infty} e^{-\alpha x} d x \int_{0}^{\infty} e^{-\beta I} d I P_{0}\left[Q(x)=0, \int_{0}^{x} Q^{2}=I\right] \\
& =\sqrt{\frac{m}{\pi}} \int_{0}^{\infty} \frac{e^{-\alpha x} d x}{\sqrt{\operatorname{sh} m x}} \text { with } m=\sqrt{2 \beta}=q e^{\sqrt{-1} \pi / 4} \\
& =\frac{1}{\sqrt{\pi m}} \int_{0}^{\infty} e^{-(p / q) \times e^{\sqrt{-1} \pi / 4}} \frac{d x}{\sqrt{\operatorname{sh} x}}
\end{aligned}
$$

behaves like $q^{-1 / 2}$ or like $p^{-1 / 2}$ according as $p / q$ is of moderate size or $p / q \uparrow \infty$.

Now, in the language of the local time,

$$
\begin{gathered}
\mathfrak{t}(x, Q)=\lim _{\varepsilon \downarrow 0} \varepsilon^{-1} \operatorname{meas}\left(0 \leq x^{\prime} \leq x: Q \leq Q\left(x^{\prime}\right)<Q+\varepsilon\right), \\
\widehat{I I}=E_{Q^{\prime}}\left[\int_{0}^{\infty} e^{-\alpha L} e^{-\beta \int_{0}^{L} Q^{2}} d \mathfrak{t}(L, Q)\right] .
\end{gathered}
$$

This may be put into a convenient form by cutting up the half-line $0 \leq L<\infty$ according to the passage time $L_{0}=\min [x: Q(x)=0]$ and the succeeding "loop times" $L_{1}<L_{2}<$ etc. depicted in Fig. 1. Write $I(x)=\int_{0}^{x} Q^{2}$ and $I_{n}=I\left(L_{n}\right)$ for $n=0,1,2,3$, etc. Then

$$
\begin{aligned}
\widehat{I I}= & E_{Q^{\prime}}\left[\int_{0}^{L_{0}} e^{-\alpha x} e^{-\beta I(x)} d \mathfrak{t}(x, Q)\right] \\
& +E_{Q^{\prime}}\left[e^{-\alpha L_{0}-\beta I_{0}}\right] \times \sum_{n=0}^{\infty} E_{0}\left[e^{-\alpha L_{n}-\beta I_{n}}\right] \times E_{0}\left[\int_{0}^{L_{1}} e^{-\alpha x-\beta I} d \mathfrak{t}\right] \\
= & E_{Q^{\prime}}\left[\int_{0}^{L_{0}} e^{-\alpha x-\beta I} d \mathfrak{t}\right]+\frac{E_{Q^{\prime}}\left[\int_{L_{0}}^{L_{1}} e^{-\alpha x-\beta I} d \mathfrak{t}\right]}{1-E_{0}\left[e^{-\alpha L_{1}-\beta I_{1}}\right]} \\
= & \widehat{I I I}+\widehat{I V},
\end{aligned}
$$

in which the independence of loops was used to reduce the sum in line 2 to a geometrical series, and the outlying factors, representing the initial passage from $Q^{\prime}$ to 0 and the final passage from 0 to $Q$, were combined to produce $E\left[\int_{L_{0}}^{L_{1}}\right.$ etc. $\left.d \mathfrak{t}\right]$. Now the technical Items $2,3,4$ show that $\widehat{I I I}$ is of class $C^{1}\left(\sqrt{-1} R^{2}\right)$, so the same is true of the product $\widehat{I} \widehat{I I I}$, with the added feature that this product and its gradient 
are summable. It follows that

$$
I * I I I=-\frac{1}{4 \pi^{2}} \int_{\sqrt{-1} R^{2}} e^{\alpha L+\beta N} \widehat{I I I}=O\left(L^{-1}\right),
$$

this estimate being independent of $Q Q^{\prime}$ in the samll, as you will readily check. The same remarks apply, in part, to $\widehat{I I V}$, only now the inverse transform must be taken as in Fig. 2a to avoid the root of $e(\alpha, \beta)=E_{0}\left[e^{-\alpha L_{1}-\beta I_{1}}\right]=1$ at $\alpha=\beta=0$; see Item 5. Now comes the descent.

Fig. 2

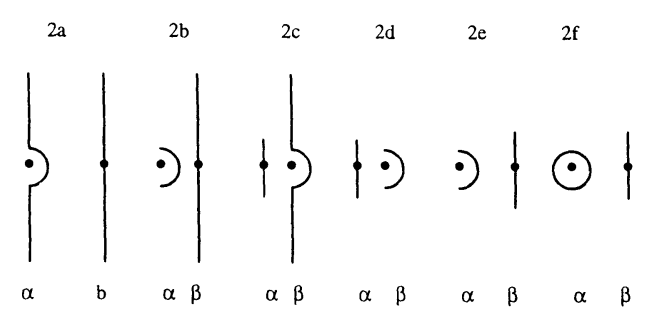

Step 1 removes the vertical $\alpha$-segments in Fig. 2a with an error of magnitude $L^{-1}$, as above, leaving the integral depicted in Fig. 2b. Step 2 distorts Fig. 2 b into Fig. 2c. Step 3 removes the vertical $\beta$-segments at like cost, leaving the integral depicted in Fig. 2d. Step 4 distorts Fig. 2 d into Fig. 2e. Now $e(\alpha, \beta)=1$ has, for small real $\beta$, a simple root $\alpha=\alpha(\beta)=-\beta D+\sigma^{2} \beta^{2} / 2+$ etc. with positive $\sigma^{2}=E_{0}\left(I_{1}-\right.$ $\left.L_{1} D\right)^{2} / E_{0}\left(L_{1}\right)$, as you will check, ${ }^{19}$ and you may take the $\beta$-segment in Fig. 2 e so short that $\alpha(\beta)$ lies inside the $\alpha$-circle seen there. Step 5 estimates the integral over the omitted left-hand arc of that with the aid of technical Items 1 and 2: if the radius $r$ is small, then the top of $\widehat{I V}$ is of magnitude $\leq E_{Q^{\prime}}\left(e^{r L_{0}}\right) E_{0}\left[e^{r L_{1}} \mathfrak{t}\left(L_{1}, Q\right)\right]$, and as this is finite, so the integral is controlled by $\int_{\pi / 2}^{3 \pi / 2} e^{r \cos \theta L} d \theta=O\left(L^{-1}\right)$. Step 6 is now permitted, which is to integrate as in Fig. $2 \mathrm{f}$, evaluating the $\alpha$-integral as $2 \pi \sqrt{-1} \times$ the residue at the pole $\alpha(\beta)=-\beta D+\sigma^{2} \beta^{2} / 2+$ etc.: up to errors of magnitude $L^{-1}$,

$$
\begin{aligned}
I * I V & =-\frac{1}{4 \pi^{2}} \int_{\phi} e^{\beta N} d \beta \int_{\odot} e^{\alpha L} d \alpha \widehat{I} \cdot \widehat{I V} \\
& =-\frac{1}{4 \pi^{2}} \int_{\phi} e^{\beta N} d \beta 2 \pi \sqrt{-1} e^{-L D \beta+L \sigma^{2} \beta^{2} / 2+\text { etc }} \widehat{I}(0) \frac{E_{0}\left[\mathfrak{t}\left(L_{1}, Q\right)\right]}{E_{0}\left(L_{1}\right)}+o(1),
\end{aligned}
$$

in which the $o(1)$ is controlled by the size of the $\beta$-segment. But $N=D L$, so the $\beta$-integral is nothing but

$$
\frac{1}{2 \pi \sqrt{-1}} \int_{-\sqrt{-1} \infty}^{\sqrt{-1} \infty} e^{L \sigma^{2} \beta^{2} / 2} d \beta=\frac{1}{\sqrt{2 \pi \sigma^{2} L}}
$$

$\overline{19-E_{0}\left(L_{1}\right)}=\partial e / \partial \alpha$ at $\alpha=\beta=0$ does not vanish. $\alpha^{\prime}(0)=-E_{0}\left(I_{1}\right) / E_{0}\left(L_{1}\right)=-D$ 
up to an exponentially small error; also, $E_{0}\left[\mathfrak{t}\left(L_{1}, Q\right)\right] / E_{0}\left(L_{1}\right)=\psi_{0}^{2}(Q)$, as you may check either by the technical Item 2 or by the law of large numbers; ${ }^{20}$ and all this estimation is independent of $Q Q^{\prime}$ in the small, so that the density $I * I I$ in the top of the micro-canonical mean can be replaced by

$$
\int_{0}^{\infty} d L^{\prime} \int_{0}^{\infty} d N^{\prime} E_{Q}\left[H, Q\left(L^{\prime}\right)=Q^{\prime}, \int_{0}^{L^{\prime}} Q^{2}=N^{\prime}\right] A\left(L^{\prime}\right) B\left(N^{\prime}\right) \frac{\psi_{0}^{2}(Q)}{\sqrt{2 \pi \sigma^{2} L}} .
$$

You can even remove the $B$ and integrate over all $Q Q^{\prime} \in R^{2}$, at the cost of a small error relative to $Z$, and so obtain

$$
M_{N / L=D}(H) \cong \frac{\int_{-\infty}^{\infty} \psi_{0}^{2}(Q) E_{Q}(H) \times\left(2 \pi \sigma^{2} L\right)^{-1 / 2}}{Z=\int_{-\infty}^{\infty} \psi_{0}^{2}(Q) E_{Q}(1) \times\left(2 \pi \sigma^{2} L\right)^{-1 / 2}}=M_{\infty}(H)
$$

the same principles having been applied to the bottom as to the top. The proof is finished.

\section{Martin Boundaries and the Local Limit Theorem}

Amateurs of the Martin boundary ${ }^{21}$ will recognize the ratio

$$
Z^{-1} P_{Q^{\prime}}\left[Q\left(L-L^{\prime}\right)=Q, \int_{0}^{L^{\prime}} Q^{2}=N-N^{\prime}\right]:
$$

assuming it has a limit $h\left(L^{\prime}, Q^{\prime}, N^{\prime}\right)$ for suitable, $L \uparrow \infty, N \uparrow \infty$, and $Q$, you expect this function to solve

$$
0=\left[\frac{\partial}{\partial L^{\prime}}+\frac{1}{2} \frac{\partial^{2}}{\partial Q^{\prime 2}}+m\left(Q^{\prime}\right) \frac{\partial}{\partial Q^{\prime}}+\left(Q^{\prime}\right)^{2} \frac{\partial}{\partial N^{\prime}}\right] h=\left(\frac{\partial}{\partial L^{\prime}}+\mathfrak{G}_{+}\right) h .
$$

Now for fixed $1 \leq L^{\prime}<\infty, L \uparrow \infty$, and general $N / L=D$, the micro-canonical mean

$$
\begin{aligned}
M_{N / L=D}(H)= & \int E_{Q}\left[H, Q\left(L^{\prime}\right)=Q^{\prime}, \int_{0}^{L^{\prime}} Q^{2}=N^{\prime}\right] \\
& \times Z^{-1} P_{Q^{\prime}}\left[Q\left(L-L^{\prime}\right)=Q, \int_{0}^{L-L^{\prime}} Q^{2}=N-N^{\prime}\right]
\end{aligned}
$$

\footnotetext{
$20 E_{0}\left[\mathfrak{t}\left(L_{1}, Q\right)\right] / E_{0}\left(L_{1}\right)=\lim _{L \uparrow \infty} L^{-1} \int_{0}^{L} p(x, 0, Q) d x=\psi_{0}^{2}(Q)$

21 See Williams [1979] for such matters
} 
tends to the petit mean $M_{\infty}^{*}(H)$ corresponding to $F^{*}(Q)=F(Q)+c Q^{2}, c$ being adjusted so that $M_{\infty}^{*}\left(Q^{2}\right)=D$. The latter can be expressed as

$$
\begin{aligned}
M_{\infty}^{*}(H)=\int & E_{Q}\left[H, Q\left(L^{\prime}\right)=Q^{\prime}, \int_{0}^{L^{\prime}} Q^{2}=N^{\prime}\right] \\
& \times \psi_{0}^{*}\left(Q^{\prime}\right) \psi_{0}^{*}(Q) \frac{\psi_{0}(Q)}{\psi_{0}\left(Q^{\prime}\right)} e^{-L N^{\prime}} e^{\left(\lambda_{0}^{*}-\lambda_{0}\right) L^{\prime}},
\end{aligned}
$$

since the density $d P_{Q}^{*} / d P_{Q}$ restricted to the field of $Q(x): 0 \leq x \leq L^{\prime}$ is

$$
\begin{aligned}
\frac{\exp \left[\int_{0}^{L^{\prime}} m^{*} d Q-\frac{1}{2} \int_{0}^{L^{\prime}}\left(m^{*}\right)^{2}\right]}{\exp \left[\int_{0}^{L^{\prime}} m d Q-\frac{1}{2} \int_{0}^{L^{\prime}} m^{2}\right]} & =\frac{\exp \left[\int_{0}^{L^{\prime}} d \lg \psi_{0}^{*}(Q)-\int_{0}^{L^{\prime}} F^{*}(Q)+\lambda_{0}^{*} L^{\prime}\right]}{\exp \left[\int_{0}^{L^{\prime}} d \lg \psi_{0}(Q)-\int_{0}^{L^{\prime}} F(Q)+\lambda_{0} L^{\prime}\right]} \\
& =\frac{\psi_{0}^{*}\left(Q^{\prime}\right)}{\psi_{0}^{*}(Q)} \frac{\psi_{0}(Q)}{\psi_{0}\left(Q^{\prime}\right)} e^{-c \int_{0}^{L^{\prime}} Q^{2}} e^{\left(\lambda_{0}^{*}-\lambda_{0}\right) L^{\prime}},
\end{aligned}
$$

in which $Q=Q(0)$ and $Q^{\prime}=Q\left(L^{\prime}\right)$, so for fixed $Q, L \uparrow \infty$, and $N / L=D$, you expect that

$$
\begin{gathered}
\lim _{L \uparrow \infty} Z^{-1} P_{Q^{\prime}}\left[Q\left(L-L^{\prime}\right)=Q^{\prime}, \int_{0}^{L-L^{\prime}} Q^{2}=N-N\right] \\
=\psi_{0}^{*}\left(Q^{\prime}\right) \psi_{0}^{*}(Q) \frac{\psi_{0}(Q)}{\psi_{0}\left(Q^{\prime}\right)} e^{-c N^{\prime}} e^{\left(\lambda_{0}^{*}-\lambda_{0}\right) L^{\prime}} \equiv h .
\end{gathered}
$$

This is the "local limit/renewal theorem"; compare Feller [1971]. The function $h$ is, in fact, a solution of $\left(\partial / \partial L^{\prime}+\mathfrak{G}_{+}\right) h=0$, and the formula is perfectly correct: indeed, the results of Krylov [1987] show that the functions $Z^{-1} P_{Q^{\prime}}$ [etc.] form a locally compact family and, to identify the limit unambiguously, you have only to choose $H$ to be a general test function in the variables $Q, Q^{\prime}$, and $N^{\prime}, L^{\prime}$ being fixed. These remarks suggest that it may be interesting to study the full Martin boundary of the space-time diffusion $x \rightarrow\left[x, Q(x), I(x)=\int_{0}^{x} Q^{2}\right]$ with infinitesimal operator $\partial / \partial x+\mathfrak{G}_{+}=\partial / \partial x+\frac{1}{2} \partial^{2} / \partial Q^{2}+m \partial / \partial Q+Q^{2} \partial / \partial I$. This is left to the future except to note that for the Ornstein-Uhlenbeck process with mass $m$, the minimal space-time functions are of the form

$$
h=\exp \left[\alpha Q e^{m^{\prime} x}-\frac{\alpha^{2}}{2} \frac{e^{2 m^{\prime} x}-1}{2 m^{\prime}}-\beta x+\beta \gamma^{2}-\gamma I / 2\right]
$$

with $\gamma \geq-m^{2}, 2 \beta=m \pm \sqrt{\gamma+m^{2}}$, and either $\alpha=0$ or else $m^{\prime}=$ $\mp(1 / 2) \sqrt{\gamma+m^{2}}$; in particular, the boundary is a topological plane. Note that the function $h$ is more general than the "micro-canonical" functions suggested by the thermodynamic limit; the latter arise precisely for $\alpha=0$. It is natural to conjecture that the general boundary is similar, but nothing more is known about it. 


\section{Gibbs' Principle}

Let $P_{0}$ be the law of the original stationary diffusion of the petit canonical ensemble for $L=\infty$, and let $P$ be the law of any other stationary process (Markovian or not). Let $\Delta$ be the density of $P$ with respect to $P_{0}$, both restricted to the field of $Q(x): 0 \leq x \leq L$. The rate of entropy production of $P$ relative to $P_{0}$ is

$$
h=\lim _{L \uparrow \infty}-\frac{1}{L} \int \Delta \lg \Delta d P_{0}=\lim _{L \uparrow \infty}-\frac{1}{L} \int \lg \Delta d P
$$

$h \leq 0$ in any case, with the understanding that $h=-\infty$ if $\Delta$ does not make sense. Gibbs principle ${ }^{22}$ asserts that, in the class of all laws $P$ with fixed mean square $\int Q^{2} d P=D$, the micro-canonical ensemble makes $h$ biggest. This is easy to prove. ${ }^{23}$ Let $\Delta_{*}$ and $h_{*}$ be the corresponding objects with the micro-canonical ensemble $P_{*}$ in place of $P_{0}$. Then

$$
h-h_{*}=\lim _{L \uparrow \infty} \frac{1}{L} \int \lg \frac{\Delta_{*}}{\Delta} d P
$$

is independent of $P$ : in fact, using for reference the free Brownian motion with starting point distributed by the infinite measure $d h$, the density of $P_{0}$ is ${ }^{24}$

$$
\begin{aligned}
\psi_{0}^{2}(Q) e^{\int m_{0} d Q-(1 / 2) \int m_{0}^{2}} & =\psi_{0}^{2}(Q) e^{\int d \lg \psi_{0}} e^{-\int\left(F-\lambda_{0}\right)} \\
& =\psi_{0}(Q) \psi_{0}\left(Q^{\prime}\right) e^{-\int\left(F-\lambda_{0}\right)} \quad \text { with } \quad Q^{\prime}=Q(L),
\end{aligned}
$$

so

$$
\frac{\Delta_{*}}{\Delta}=\frac{d P_{0}}{d P_{*}}=\frac{\psi_{0}(Q) \psi_{0}\left(Q^{\prime}\right)}{\psi_{*}(Q) \psi_{*}\left(Q^{\prime}\right)} e^{-c \int Q^{2}+\left(\lambda_{0}-\lambda_{*}\right) L}
$$

and

$$
\begin{aligned}
\int \lg \frac{\Delta_{*}}{\Delta} d P & =2 \int \lg \frac{\psi_{0}(Q)}{\psi_{*}(Q)} d P-c D L+\left(\lambda_{0}-\lambda_{*}\right) L \\
& =O(1)+L\left(\lambda_{0}-\lambda_{*}-c D\right) .
\end{aligned}
$$

But then it is the same to maximize $h_{*}$ as to maximize $h$, and it is easy to see that $h_{*}<0$ unless $\Delta_{*}=1$, i.e., unless $P=P_{*}$.

\section{References}

Berlin, T.H., Kaç, M.: The spherical model of a ferromagnet. Phys. Rev. 86, 821-835 (1952)

Cameron, R., Martin, W.T.: Transformation of Wiener integrals under a general class of transformations. TAMS 58, 184-219 (1945)

Döblin, W.: Sur les propriétés asymptotiques de mouvement régis par certains types de chaines simples. Bull. Math. Roum. Sci. 39, No. 1, 57-115, No. 2, 3-61 (1937)

Dobrushin, R.L., Tirozzi, B.: The central limit theorem and the problem of equivalence of ensembles. Commun. Math. Phys. 54, 173-192 (1977)

22 Gibbs [1902]

23 Varadhan showed us this nice way

24 The integral $\int$ is extended from $x=0$ to $x=L$ 
Feller, W.: Introduction to probability theory and its applications (2). 2nd ed., New York: Wiley 1971

Gibbs, J.M.: Elementary principles in statistical mechanics, New Haven, CT: Yale U. Press 1902; New York: Dover 1960

Girsanov, I.V.: On transforming a certain class of stochastic processes by absolutely continuous substitution of measures. Teor. Veroyatnost 5, 314-330 (1960)

Hörmander, L.: Hypoelliptic differential operators of second order. Acta Math. 119, 147-171 (1967)

Itô, K., McKean, H.P.: Diffusion processes and their sample paths. Berlin, Heidelberg, New York: Springer 1965

Krylov, N.V.: Nonlinear elliptic and parabolic equations of second order. Dordrecht, Boston, Lancaster, Tokyo: Reidel 1987

Kolmogorov, A.N.: Local limit theorem for classical Markov chains. Izvest. Akad. Nauk SSR 13, 281-300 (1949)

Lebowitz, J.L., Rose, H.A., Speer, E.R.: Statistical mechanics of the nonlinear Schrödinger equation. J. Stat. Phys. 50, 657-687 (1988)

McKean, H.P.: Stochastic integrals. New York: Academic Press 1969

McKean, H.P., Vaninsky, K.L.: Statistical mechanics of nonlinear wave equations. 1993 (1), to appear

McKean, H.P., Vaninsky, K.L.: Gibbsian invariant measures for NLS. 1993 (3), to appear

Molchanov, S.A., Sudarev, J.N.: Gibbs states in the spherical model. Sov. Math. Dokl. 16, 1254-1257 (1975)

Williams, D.: Diffusions, Markov processes, and martingales. Vol. 1, New York: Wiley 1979

Communicated by A. Jaffe 